\title{
SCIDiC
}

\author{
International Journal of Dentistry and Oral Science (IJDOS) \\ ISSN: $2377-8075$
}

\section{The Association Between Stage, Grade and Cervical Node Metastasis In Oral Cancer - A Single Institute Retrospective Study}

Research Article

Mahathi Neralla ${ }^{1 *}$, Sunil Akula², Rinku George ${ }^{3}$

${ }^{1}$ Reader, Saveetha Oral Cancer Institute, Department of Oral and Maxillofacial Surgery, Saveetha Dental College, 162, Poonamallee High Road, Velappanchavadi, Chennai, Tamil Nadu, India.

${ }^{2}$ Fellow, Saveetha Oral Cancer Institute, Department of Oral and Maxillofacial Surgery, Saveetha Dental College, 162, Poonamallee High Road, Velappanchavadi, Chennai, Tamil Nadu, India.

${ }^{3}$ Professor, Saveetha Oral Cancer Institute, Department of Oral and Maxillofacial Surgery, Saveetha Dental College, 162, Poonamallee High Road, Velappanchavadi, Chennai, Tamil Nadu, India.

\section{Abstract}

Introduction: OSCCs have a peculiar reputation for their affinity to the lymphatics and are notorious for spreading to deep cervical lymphatics. It is a well known fact by now that the mere presence of cervical metastasis would reduce the 5 year survival rate by an astonishing 50 percent. There is still some persisting controversy in this crucial area of treatment planning of a clinically $\mathrm{N} 0$ neck. From a wait and watch policy to doing a sentinel biopsy to performing an elective neck dissection, the opinions are changing by the day.

Aims and objective: The Aim of the study was to see the presence of any association between clinical and pathological indicators and cervical metastasis in patients having Oral Squamous Cell Carcinoma.

Materials and methods: Retrospective analysis of case sheets and post operative histopathology reports of patients operated in our College from 2015-2017 were done and all the data related to cervical metastasis was noted. We classified cervical metastasis in to two groups: positive for cases with histological evidence of cervical metastasis and negative for those with no histological evidence. All patients were clinically staged according to the AJCC 7th edition guidelines. Known clinical and pathological indicators, in addition to the clinical staging were analysed to find out if they were predictive of neck disease using the chi square test. The survival time was calculated using Kaplan-Meier survival curve only wherever necessary. The log rank test was used to detect the differences for survival time. We compared this data with the previously available literature. Results: The likelihood of performing an MRND with a higher $\mathrm{T}$ and $\mathrm{N}$ stage is statistically significant with a $\mathrm{p}$ value of $<$ 0.001. There was no significant association between TNM staging, histological grade and the presence of cervical metastasis. Though the survival is more in patients with no nodal disease, it is statistically not significant $(p=0.620)$ in Chi square test and in Kaplar-Meier analysis $(p=0.340)$. A similar result was obtained for disease free survival analysis also $(p=0.115$ in Chi square test and $\mathrm{p}=0.081$ in Kaplar-Meier analysis).

Conclusion: There seems to be no significant association between known clinical and pathological indicators and the likelihood of a patient having cervical metastasis.

Keywords: Cervical Metastasis; R0 Resection; Clearance; Clinical Staging.

\section{Introduction}

Oral squamous cell carcinoma (OSCC) is a major contributor in the realm of head and neck cancers with oral cavity being the most common site of malignant tumors of the head and neck. The most common malignant variant in the oral cavity is squamous cell carcinoma. Oral squamous cell carcinoma (OSCC) is frequently associated with poor prognosis even with T1 and T2

\section{*Corresponding Author:}

Mahathi Neralla,

Reader, Saveetha Oral Cancer Institute, Department of Oral and Maxillofacial Surgery, Saveetha Dental College, 162, Poonamallee High Road, Velappanchavadi, Chennai, Tamil Nadu, India.

Email Id: nerallamahathi@gmail.com

Received: March 16, 2021

Accepted: April 02, 2021

Published: April 05, 2021

Citation: Mahathi Neralla, Sunil Akula, Rinku George. The Association Between Stage, Grade and Cervical Node Metastasis In Oral Cancer - A Single Institute Retrospective Study. Int J Dentistry Oral Sci. 2021;08(04):2230-2235. doi: http://dx.doi.org/10.19070/2377-8075-21000441

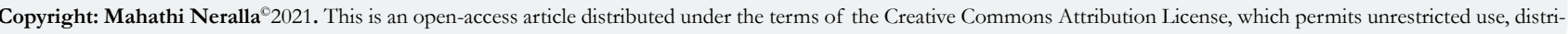
bution and reproduction in any medium, provided the original author and source are credited. 
sized lesions, as OSCC carries a high risk of cervical lymph node metastasis. Treatment of N0 neck in patients is controversial [1]. Amongst the various prognostic factors, cervical metastasis is the most important one. Various studies have given a micro-metastasis rate of 15 to $20 \%$ depending on the type of subsite [2]. While each sub-site has its own significant role in cervical metastasis, the size of the lesion also influences their capability to metastasize [2]. There is considerable evidence on histological differentiation playing a role in tumour activity. There are various investigations that aid in the detection of cervical metastasis with a reasonable amount of reliability and each one has its own pros and cons, the discussion of which is beyond the scope of this study. Though techniques like the sentinel node biopsy have become popular due to their ability to stage the neck and thus avoid surgical morbidity from a neck dissection, it's role is unclear in the field of oral cancer. FNAC has a high specificity, but its sensitivity is relatively low in detecting cervical metastasis and hence it cannot be relied upon when taking a major decision, such as whether or not to do a neck dissection. It was previously found that even the most sophisticated detection technique might not be able to reveal occult metastases $[3,4]$ and taking in to account only the CT and USG findings in detecting positive nodes in the neck [5], one can be wrong by 20 percent. Adequate treatment of cervical lymph nodes ensures loco-regional control of the disease [1].

The most recent AJCC staging system released in 2018 has had significant changes from its previous editions. The clinical staging however does not consider some crucial factors like histological grade and patient demographics to name a few. Though a nomogram based predictor score is superior to the conventional TNM staging, it still would be a considerable time when we would step beyond the TNM system [6].

Though continuous efforts are being made by various researchers to find a less invasive method in order to reduce the morbidity from extensive neck procedures, in the present scenario, the conventional remains to be gold standard. The neck dissection as a procedure, though invasive, can be tailored accordingly on a case by case basis to avoid most of the ills it is considered to bring upon the patients. The valuable information this procedure gives, does not only just help in determining the prognosis of the disease but also aids the clinician in taking crucial decisions like giving a combined modality treatment by including post-operative radiotherapy and chemotherapy. Elective neck dissection (END) gives us better results than the "wait and see" approach in terms of survival rate [7].

The Aim of the study was to see the presence of any association between known clinical and pathological indicators and cervical metastasis in patients having Oral Squamous Cell Carcinoma.

\section{Materials and methods}

This Retrospective study included patients who were treated at the Department of Oral Oncology and Reconstructive Services of a Private Dental College, Chennai. Medical records of patients operated in our Institute from June 2015- April 2020 were reviewed. All the required data like demographic details, age, sex and type of surgery performed were noted. Clinical staging was noted along with the lymph node status before and after surgery. Post-operative histopathological reports were reviewed and cases with neck metastasis were noted as positive and those without metastasis were noted as being negative. Clinical and pathological $\mathrm{N}$ status was noted. The Institutional Ethics Committee approved the study (SRB/SDC/FACULTY/20/OSURG/02).

\section{Inclusion Criteria}

Patients with histologically proven squamous cell carcinoma of the oral cavity.

Patients not previously treated else where.

Patients for whom surgery was the first option and was done as their initial treatment.

\section{Exclusion Criteria}

Patients not fit for surgery and presence of other contraindications for surgery (inoperable or unresectabletumors ).

Presence of distant metastasis or local recurrence at the time of admission.

Presence of other simultaneous primary tumors or conditions that precludes surgery.

Patients managed with preoperative radiotherapy and neoadjuvant chemotherapy.

In the clinical examination, history sheets were used where all the patient information and relevant history were filled. Primary tumor size and location (subsite) and lymph node involvement were evaluated through a physical examination, CT scan, MRI routinely and PET-CT scan (where relevant). All the details of the clinical examination done were noted down along with the clinical staging. The American Joint Committee on Cancer (AJCC- 7th edition) designated staging by TNM Classification was used. In the histopathological report tumours were graded as well differentiated, moderately differentiated and poorly differentiated. The necks with lymph nodes which harbored tumour cells were labeled as 'positive'. Necks with any other pathology like inflammatory/reactive nodes other than tumour infiltrates were labeled as negative.

Primary tumor resection was done with an oncologic safety margin of greater than $1 \mathrm{~cm}$ in all tumor sites. Either selective neck dissection (SND; level I, II, III) or MRND (level I, II, III, IV, V) was performed, depending on neck involvement and tumor size.

Intraoperatively frozen section was done for evaluation of the resection margins and revised according to results obtained. Postoperatively, the resected specimen was sent for biopsy for detailed examination, and for a report on the invasion of the extracapsular lymph nodes in particular. The biopsy specimens were labeled with neck levels and sublevels of dissection and were immersed in $10 \%$ buffered formalin and sent for examination. The clinical N0 or $\mathrm{N}+$ status was based on TNM staging and pathological $\mathrm{pN} 0$ or $\mathrm{pN}+$ status were determined from the postoperative biopsy results. Adjuvant radiotherapy was performed in patients according to our Institute criteria:

\section{Presence of positive nodes.}


2. If depth of invasion greater than $10 \mathrm{~mm}$.

3. Presence of close (less than $5 \mathrm{~mm}$ ) and positive margins.

4. When $\mathrm{T}$ stage is more,

5. Any extarcapsular spread.

6. Perineural invasion.

Radiotherapy was initiated within 4 to 6 weeks after surgery if necessary, according to the patient's condition. Follow up of the postoperatively was done once every 4 weeks for the first year and once every 2 months in the second year.

\section{Statistical Methods}

All statistical analyses were performed with SPSS 20.0 version. The primary outcome variable was presence of cervical metastasis. The secondary outcome variables were the relation between neck node metastasis and clinical and pathological indicators like stage and histological grade. The association of predictors with cervical metastasis and overall survival and disease free survival was done using chi- square test. The survival time was calculated using Kaplan-Meier survival curve only wherever applicable. The $\log$ rank test was used to detect the differences for survival time. The $\mathrm{p}<0.05$ was considered significant.

\section{Results}

Of 95 patients, $71(74.73 \%)$ were men and 24 (25.26\%) were women; the number of patients with $<50$ age at diagnosis was 42 $(44.21 \%)$ and $>50$ was $53(55.78 \%)$. The basic characteristics of the patients are shown in Table 1 .

\section{Subsites Distribution}

According to the subsite, buccal mucosa and gingivobuccal sulcus lesion contributed to the majority of cases [36 (37.89\%)] followed by oral tongue and floor of the mouth lesions which accounted for 27 (28.42\%) and retromolartrigone (RMT) 17 (17.89\%) cases. The number of pure alveolus cases were $8(8.4 \%)$, palate and maxilla cases were $7(7.36 \%)$ respectively.

Table 1. The basic characteristics of the patients in our study.

\begin{tabular}{|c|c|}
\hline Mean Age (years) & $53 \pm 11.9$ in years \\
\hline Gender & Number of patients \\
\hline Male & $24(74.73 \%)$ \\
\hline Female & Number of patients \\
\hline Neck Dissection & $35(36.84 \%)$ \\
\hline SND & $56(58.94 \%)$ \\
\hline MRND & Number of patients \\
\hline Adjuvant therapy & $43(45.26 \%)$ \\
\hline Yes & $52(54.73 \%)$ \\
\hline No & Number of patients \\
\hline Lesion Site & 34 \\
\hline ALV & 27 \\
\hline BM+GBS & 7 \\
\hline LT + FOM & 17 \\
\hline PT+MAX & \\
\hline RMT & \\
\hline
\end{tabular}

Table 2. The table 2 depicts the association between the clinical $\mathrm{T}$ stage and $\mathrm{N}$ stage with respect to the type of neck dissection performed.

\begin{tabular}{|c|c|c|c|}
\hline & SND & MRND & \\
\hline & $\mathbf{n} \mathbf{( \% )}$ & $\mathbf{n ~ ( \% )}$ & p-value \\
\hline T-stage & & & \\
\hline 1 & 13 & 3 & $<0.001$ \\
\hline 2 & 15 & 18 & \\
\hline 3 & 3 & 13 & \\
\hline 4 & 3 & 22 & \\
\hline N-stage & & & \\
\hline 0 & 28 & 15 & $<0.001$ \\
\hline 1 & 3 & 24 & \\
\hline 2 & 3 & 17 & \\
\hline
\end{tabular}




\section{Type Of Neck Dissection}

Out of the 95 cases MRND was performed in $56(58.94 \%)$ of patients and SND was performed in $35(36.84 \%)$ of patients. In 4 cases $(4.21 \%)$ neck dissection was not performed.

Out of 16 patients diagnosed with T1 lesion, we performed SND in 13 patients and MRND in 3 patients, out of 33 T2 patients, we performed SND in 33 and MRND in 15, out of 16 T3 cases, we performed SND in 3 and MRND in 13 of them and in 24 T4 cases, we performed SND in 3 while in the majority 22, we performed MRND. The likelihood of performing an MRND with a higher T stage is statistically significant with a $\mathrm{p}$ value of $0.001(<$ 0.05) (Table 2).

A similar trend was noticed when comparing the clinical $\mathrm{N}$ stage to the type of neck dissection. Out of $43 \mathrm{~N} 0$ patients, we performed SND in 28 of them and MRND in 15 patients. While in $27 \mathrm{~N} 1$ cases and $20 \mathrm{~N} 2$ cases, we performed SND in in 3 of each, and MRND in $24 \mathrm{~N} 1$ cases and $17 \mathrm{~N} 2$ cases. This trend was also statistically significant with a p value of $0.001(<0.05)$ (Table 2).

\section{Neck Stages Distribution}

On clinical examination, $49(51.57 \%)$ had been staged having nodal disease.

\section{Adjuvant Therapy Distribution}

$43(45.26 \%)$ patients received adjuvant therapy in the form of radiotherapy and chemotherapy and $52(54.73 \%)$ of them had not received either radiotherapy or chemotherapy.

\section{Significance Of Clinical Staging and Surgical Margin On Cervical Metastasis}

When clinical $\mathrm{T}$ stage and pathological $\mathrm{N}$ stage were compared, the neck was negative for cervical mets in 10 of $16 \mathrm{~T} 1,20$ of 35 T2, 10 of 16 T3 and 27 T4 cases while 6 T1, 15 T2, 6 T3 and 12 T4 cases had a positive neck. No significant trend was observed. A total of 39 cases were positive for neck disease $(p=0.954)$ (Fig1).

While comparing clinical $\mathrm{N}$ stage and pathological $\mathrm{N}$ stage, out of 45 cases diagnosed with an N0, 31 were free of neck disease while 14 were positive for cervical metastasis. Out of $28 \mathrm{~N} 1$ cases and $21 \mathrm{~N} 2$ cases, $14 \mathrm{~N} 1$ and $10 \mathrm{~N} 2$ were negative while $14 \mathrm{~N} 1$ and $11 \mathrm{~N} 2$ cases were positive for neck disease. The trend shows that detection of clinically enlarged cervical lymph nodes increases the likelihood of having a true nodal disease by about 50 percent. But it is not statistically significant $(\mathrm{p}=0.145)(\mathrm{Fig} 2)$.

When the overall stage was considered, out of 11 stage 1 patients, 4 were positive for nodal disease, out of 32 stage 2 patients, 7 were positive, out of 26 stage 3 patients, 12 of them were positive and out of 35 stage 4 patients, 16 of them had neck disease. There was no statistical significance seen in these findings $(\mathrm{p}=0.700)$ (Fig 3).

When histopathological grade of the lesion was considered, out of 77 cases with WDSCC, 31 had evidence of nodal metastasis.

Figure 1. Association between T stage and presence (red bar) and absence (blue bar) of metastasis. No statistically significant association $(p=0.954)$ found, which means the presence of cervical metastasis is not related to $T$ stage.

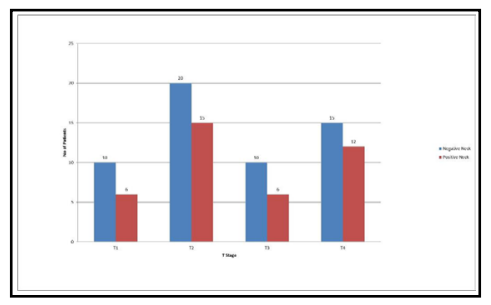

Figure 2. Association between N stage and presence (red bar) and absence (blue bar) of metastasis. No statistically significant association $(p=0.145)$ found, which means the presence of cervical metastasis is not related to $\mathrm{N}$ stage.

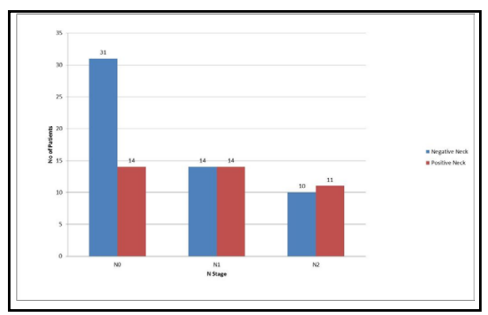

Figure 3. Association between overall stage and presence (red bar) and absence (blue bar) of metastasis. No statistically significant association $(p=0.700)$ found, which means the presence of cervical metastasis is not related to overall stage.

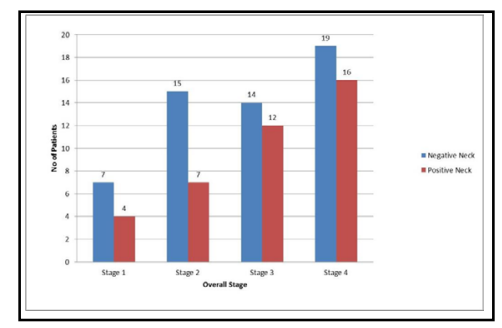


Out of 11 patients with MDSCC and 2 patients with PDSCC, 11 and 1 were positive for neck disease respectively. In the others category which included verrucous $\mathrm{CA}$ and CA cuniculattum, out of 10 patients, only 1 was positive for nodal disease/Though this finding is statistically not significant, patients with the milder form of carcinoma were less likely to have a cervical node metastasis (Fig 4).

When considering overall survival, out of the 39 patients who had cervical metastasis, 26 were alive and 13 died. Out of the 56 patients who were negative for neck disease, 40 were alive and 16 died. Though the survival is more in patients with no nodal disease, it is statistically not significant $(p=0.620)$ in Chi square test and in Kaplar-Meier analysis $(p=0.340)$. The median overall survival time for patients with negative neck was 34.967 months (Fig 5).

Out of 39 patients with cervical metastasis, 21 had recurrence of disease after surgery and 18 had no event until the last follow up. Out of 56 patients who had no nodal disease initially, 21 had recurrence of disease and 35 were disease free $(p=0.115)$ in Chi square test and in Kaplar-Meier analysis $(\mathrm{p}=0.081)$. The median disease free survival time for patients with negative neck was 27.4 months and for those with positive neck was 20.5 months (Fig 6).

\section{Discussion}

Oral squamous cell carcinomas have a substantial share in the head and neck cancers which in turn constitute approximately $15 \%$ of all cancers [8]. The incidence of oral cancer varies all over the world and is more common among men in the people of India. This can be attributed to the hard wired habit of pan chewing which includes betel nut and tobacco along with slaked lime. This is still in vogue, especially in the lower socioeconomic strata with ignorance and illiteracy further reinforcing this age old habit. In our study, the male to female ratio is $1: 3$ with males more prone to OSCC than females. According to the current data, the most commonly performed neck dissection in patients with Oral Cancer was modified radical neck dissection with preservation of all three structures. When in need the sternocleidomastoid was sacrificed in order to achieve sufficient visualization which is paramount for an optimal clearance of the lymphatic levels II to III. In cases of T1 and T2 lesions, in cases where microvascular reconstruction is planned, selective neck dissection including lymph node levels I to III (supraomohyoid) was the surgeon's choice of treatment. In our study the likelihood of performing an MRND with a higher $\mathrm{T}$ stage is statistically significant with a $\mathrm{p}$ value of $0.001(<0.05)$.

Figure 4. Association between histopathological grade and presence (red bar) and absence (blue bar) of metastasis. No statistically significant association $(\mathrm{p}=0.131)$ found, which means the presence of cervical metastasis is not related to histopathological differentiation.

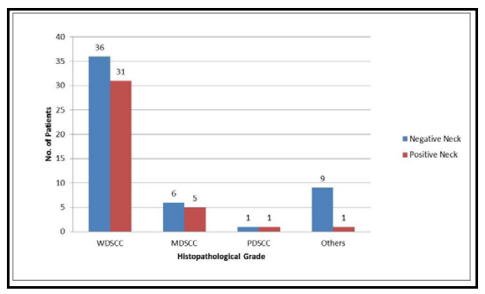

Figure 5. Kaplan-Meier survival analysis for overall survival with neck metastasis. The blue curve represents the patients with cervical metastasis while the green curve represents those patients without metastasis.

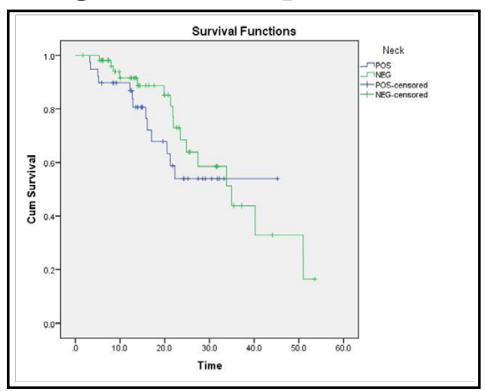

Figure 6. Kaplan-Meier survival analysis for disease free survival with neck metastasis. The blue curve represents the patients with cervical metastasis while the green curve represents those patients without metastasis.

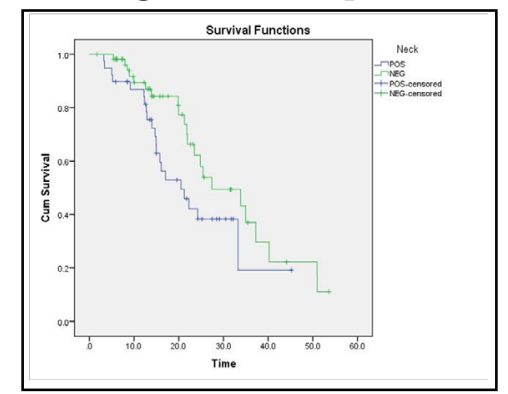


The total number of patients in our study was 95 . The mean age at presentation in our study was $53 \pm 11.9$. The mean follow up time in our study is 15 months (Inter quartile range 9.9 months to 24.8 months). In the current study we looked in to the significance of surgical margin and clinical stage in relation to cervical metastasis. The rationale for conducting this study is as follows. The lymph node status has an important impact on disease free survival (DFS) and overall survival (OS). The presence of even one positive lymph node reduces the survival by $50 \%$. We analysed the data from our college to see if it corroborates with the previous literature with regards to the factors influencing cervical metastasis.

Among the many factors like depth of invasion, growth pattern, differentiation, neutrophil/lymphocyte ratio (NLR) and the short/long axis diameter ratio ( $\mathrm{S} / \mathrm{L}$ ratio) of lymph nodes and TNM postoperative stage that were stated as important risk factors in cervical nodal metastasis in the literature [9]. We analysed the influence of histological grade and clinical staging in cervical node metastasis in patients with OSCC.

It is stated in the literature that, the larger the diameter of the tumor, the likelihood of lymph node metastasis occuring in the neck increases [9-11]. But no similar trend was observed in our study. Maybe increasing the sample size will demonstrate similar results.

Tumors with high grade (grade 3) histology, regardless of T stage, were found to present with regional metastasis more frequently. Histological grade indirectly influences regional metastasis, as achieving tumor-free margins in patients with poorly differentiated carcinomas (grade 3) is more difficult than in patients with other tumor grades [12]. But no similar trend was observed in our study. Maybe increasing the sample size will demonstrate similar results.

Histological grade was shown to have an important impact even on outcome, in a previous study done at our institution. In the previous study done in the same subset of patients, not yet published though, we found that histological grade influenced both overall and disease free survival.

The main and important casualty of the presence of cervical metastasis is locoregional recurrence. Another factor that influences prognosis and locoregional recurrence in head and neck cancer patients apart from just the presence of positive lymph nodes is lymph node ratio. Lymph node ratio is also an independent prognostic factor for locoregional recurrence and overall survival (OS). Lymph node ratio of $20 \%$ predisposes the patient to locoregional recurrence and death (prabhu 2014). Significant improvements in locoregional control have been shown in large randomized trials to significantly improve overall survival (OS) [13].

Recent studies are laying emphasis on LNR rather than merely noting the presence or absence of cervical metastasis. LNR is found to have a better prognostic value than cervical metastasis. It has been stated that survival differed drastically when LNR was taken in to account. Even with the presence of extracapsu- lar spread the impact of survival was found to be different. It is found that when LNR was less than $6 \%$, the survival benefit in patients with $\mathrm{pN} 1$ or higher is similar to those with pN0. Stratification for high-risk patients (higher than 6\% LNR) can be evaluated easily with LNR. Hence the authors of the study suggested LNR in the clinical routine [14].

\section{Conclusion}

There seems to be no significant association between known clinical and pathological indicators and the likelihood of a patient having cervical metastasis.

\section{References}

[1]. Sharma A, Kim JW, Paeng JY. Clinical analysis of neck node metastasis in oral cavity cancer. J Korean Assoc Oral Maxillofac Surg. 2018;44(6):282-8. PubmedPMID: 30637242.

[2]. Watkinson JC, Gilbert RW. Stell and Maran's textbook of head and neck surgery and oncology, 5th edn. 2012.

[3]. Ferlito A, Shaha AR, Rinaldo A, Mondin V. Management of clinically negative cervical lymph nodes in patients with malignant neoplasms of the parotid gland. Orl. 2001;63(3):123-6. PubmedPMID: 11359087.

[4]. Byers RM, El-Naggar AK, Lee YY, Rao B, Fornage B, Terry NHA, et al. Can we detect or predict the presence of occult nodal metastases in patients with squamous carcinoma of the oral tongue?. Head Neck. 1998;20(2):138-44. PubmedPMID:9484945.

[5]. Yao M, Graham MM, Hoffman HT, Smith RB, Funk GF, Graham SM, et al. The role of post-radiation therapy FDG pet in prediction of necessity for post-radiation therapy neck dissection in locally advanced head-and-neck squamous cell carcinoma. Int J RadiatOncolBiol Phys. 2004;59(4):100110.Pubmed PMID: 15234033.

[6]. Bobdey S, Sathwara J, Jain A, Saoba S, Balasubramaniam G. Squamous cell carcinoma of buccal mucosa: An analysis of prognostic factors. South Asian J Cancer.2018;7(1):49-54.Pubmed PMID: 29600236.

[7]. Haddadin KJ, Soutar DS, Oliver RJ, Webster MH, Robertson AG, MacDonald DG. Improved survival for patients with clinically T1/T2, N0 tongue tumors undergoing a prophylactic neck dissection. Head Neck. 1999;21(6):517-25. Pubmed PMID: 10449667.

[8]. SEER Cancer Statistics Review 1975-2006 - Previous Version - SEER Cancer Statistics [Internet]. SEER. 2020 [cited 4 August 2020]. Available from:

[9]. Li Y, Liu K, Ke Y, Zeng Y, Chen M, Li W, et al. Risk factors analysis of pathologically confirmed cervical lymph nodes metastasis in oral squamous cell carcinoma patients with clinically negative cervical lymph node: Results from a cancer center of central China. J Cancer. 2019;10(13):3062-9.PubmedPMID: 31281484.

[10]. Monroe MM, Gross ND. Evidence-based practice: management of the clinical node-negative neck in early-stage oral cavity squamous cell carcinoma. OtolaryngolClin North Am. 2012;45:1181-93. PubmedPMID: 22980692.

[11]. Koo BS, Choi EC, Yoon YH, Kim DH, Kim EH, Lim YC. Predictive factors for ipsilateral or contralateral central lymph node metastasis in unilateral papillary thyroid carcinoma. Ann Surg. 2009;249:840-4. Pubmed PMID: 19387316.

[12]. Shiboski CH, Schmidt BL, Jordan RCK. Tongue and tonsil carcinoma: Increasing trends in the U.S. population ages 20-44 years. Cancer. 2005;103(9):1843-9. Pubmed PMID: 15772957.

[13]. Bernier J, Cooper JS, Pajak TF, Van Glabbeke M, Bourhis J, Forastiere A, et al. Defining risk levels in locally advanced head and neck cancers: A comparative analysis of concurrent postoperative radiation plus chemotherapy trials of the EORTC (\#22931) and RTOG (\#9501). Head Neck. 2005;27(10):843-50.

[14]. Reinisch S, Kruse A, Bredell M, Lübbers HT, Gander T, Lanzer M. Is lymphnode ratio a superior predictor than lymph node status for recurrence-free and overall survival in patients with head and neck squamous cell carcinoma?. Ann SurgOncol. 2014;21(6):1912-8. Pubmed PMID:24652351. 